\title{
EL ATENEO Y LA VIDA ARTÍSTICA SEVILLANA EN 1925
}

\author{
POR GERARDO PÉREZ CALERO
}

\begin{abstract}
Este artículo analiza la relaciones entre El Ateneo de Sevilla y la vida artística de la ciudad, durante 1925. Las actividades de la "Docta Casa" en la formación de artistas, la celebración de Exposiciones, la extensión de la cultura artística, y en definitiva la variedad de aspectos en el fomento de las artes en el año anteriormente mencionado.
\end{abstract}

In this article it is analyzed the relatons on 1925 between the Ateneo of Seville and this artistic life of the city: the activities of the "Docta Casa" talking about artists formation, the celebration of Expositions, the spread of the artistical culture and definitelly the variety of aspects of foment of Arts in the City in the year mentioned before.

La interesante vida artística del Ateneo de Sevilla en relación con la ciudad desde su fundación en 1887, pasó por momentos destacados durante el primer cuarto de nuestra centuria. Es precisamente a fines de éste cuando se dan las circunstancias que creo dignas del presente trabajo, fruto, 70 años después, de intensas investigaciones que llevamos realizando desde hace algún tiempo ${ }^{1}$.

El ambiente artístico que vive Sevilla en estos momentos es consecuencia del que se había producido la década anterior como resultado a su vez de una serie de circunstancias harto complejas que se venían arrastrando en puridad desde aquella no demasiado lejana crisis del 98.

Ese ambiente de fin de siglo desemboca irremisiblemente en el fenómeno regionalista que pretende pulir la vieja esencia hispana con objeto de mostrar una mayor objetividad en la conciencia colectiva del pueblo. Se pretende por teóricos del regionalismo hacer algo nuevo aunque firmemente enraizado en la radición. Los

1. En relación con el tema puede verse mi trabajo: "Pintura andaluza y Generación del 98". Publicaciones de la Universidad de Oviedo. En prensa. 
intentos -al menos- no se harán esperar y, aunque con mayor o menor actividad, sucederán a todo los largo del primer tercio de la centuria hasta la Guerra Civil. ${ }^{2}$

En relación con éste complejo e interesante fenómeno del Regionalismo se viene escribiendo de un tiempo a esta parte con la intención de buscar sus verdaderas raíces y trascendencia mas, si desde el punto de vista arquitectónico han aparecido últimamente fecundos trabajos, la plástica artística no ha gozado de igual fortuna, siendo escasos los trabajos en este campo. ${ }^{3}$

Las artes plásticas junto a la música y las artes suntuarias, tienen en el Ateneo un interés singular, en cuya Sección de Bellas Artes no solo encuentran el lugar adecuado para su gestación sino también para su desarrollo y protección. Esta Sección, autónoma desde 1912, trece años después y bajo las presidencias sucesivas de cuatro grandes pintores hispalenses o sevillanizados (Santiago Martínez, Gustavo Bacarisas, González Santos y Félix Lacárcel) discurre su fecunda existencia con multitud de actividades que tienen como acontecimientos artísticos singulares tanto la Exposición de Primavera como la popular Cabalgata de Reyes Magos, ambas razón de ser de la misma desde 1914 y 1918 respectivamente.

Llevaba, pues, once años convocándose la Exposición que abría la estación florida en la ciudad y, como en anteriores ocasiones, la institución quiso de nuevo en 1925 volcarse con tan trascendental acontecimiento anual pues el certamen constituía además el reflejo de lo que a nivel regional se hacia en el terreno de la creación del arte contemporáneo de trascendencia nacional e incluso internacional. ${ }^{4}$

Por entonces, Sevilla, como otras importantes capitales españolas, se hallaba inmersa en los llamados "felices años 20" pues el Directorio Militar (1923-1926) presidido por Primo de Rivera había hecho posible una paz social y un relativo progreso material que propiciarían la celebración de la Exposición Iberoamericana, el primer gran hito artístico hispalense del siglo XX, cuyas obras se hallaban en marcha por entonces aunque su inauguración se retrasaría cuatro años.

En éste clima de progreso y bonanza económica, nada más comenzar el año, la Sección de Bellas Artes de la Docta Casa en su Junta General de 24 de enero a la sazón presidida por el joven pintor Santiago Martínez, aborda como tema único la convocatoria del certamen primaveral con el patrocinio del Ayuntamiento de la ciudad. Se acuerda el plazo de entrega de las obras en 20 de marzo y su inauguración el 29 del mismo mes. Igualmente, como es costumbre, para dignificar aun más la muestra, se conviene en invitar, entre otros, a los artistas Benedito, Covarsí, Capuz, Benlliure, Casas, Abarca,

2. Vid al respecto mis trabajos: "Gonzalo Bilbao y la pintura del regionalismo en Sevilla (1880-1930) en el Catálogo de la Exposición "Gonzalo Bilbao y la Pintura sevillana de su tiempo". Sevilla, 1988; así como: "Pintura andaluza y Generación del 98" (vid nota anterior).

3. Las actas de la Sección recogen el espíritu y la letra del certamen al especificar que se trata de la "celebración de una exposición Regional andaluza de Arte Moderno". (Libro de Actas de la Sección de Bellas Artes del Excmo. Ateneo de Sevilla. Sesión de 30 de mayo de 1913.

4. Junta Gral. de la S. B-A. Libro de Actas. 24 de enero de 1925. 
Rodríguez Jaldón y Wintuysen, así como, dado el carácter regional de la misma, de manera colectiva al Ateneo de Jerez y a los artistas de Cádiz.

Meses después y previa la convocatoria correspondiente se falla el concurso para la portada del catálogo de la exposición recayendo el premio, pese al voto negativo del pintor astigitano José Molleja (1900-1977), en el jovencísimo artista jerezano Teodoro Miciano Becerra (1903-1974), gran promesa del arte del grabado en Barcelona tras la Guerra Civil y a la sazón miembro del propio Ateneo. Su portada satisfacía los gustos más comunes en este tipo de trabajo por su sofisticada y ecléctica composición en la que se incluyen tanto el elemento clásico de la columna de un jónico rebuscado que sostiene presumiblemente la cabeza de Atenea, como en la ancha cenefa que circunda el rectángulo central compuesta a base del repertorio más o menos tradicional de elementos curvos de hojas y frutos que emergen de un gran cesto. Su antecedente puede hallarse en los ricos ejemplares decorativos de origen presumiblemente catalán que se encuentran grabados en marqueterías publicitarias para la revista "Bética" y, en cualquier caso, nos muestra el interés por este tipo de estampación que tanto atraía a los regionalistas sevillanos del Ateneo. Precisamente uno de ellos, José Andrés Vázquez Pérez (1884-1960) ${ }^{5}$, nombrado hijo adoptivo de Sevilla este mismo año, fue el encargado de redactar "El significado de la Exposición" como prólogo del catálogo en el que revierte la esencia del arte regionalista de la época. Dice lo siguiente: "Como obligado acorde en la sinfonía primaveral de las fiestas sevillanas, vibra cada año en los salones del Palacio de Bellas Artes la emoción de las almas ante las obras que forman la exposición organizada bajo los auspicios del Ayuntamiento por los artistas del Ateneo.

Tiene esta exposición de arte en primer término la hermosura de lo habitual y la regularidad de lo indispensable. Por ella los ojos ávidos del mundo que vienen a escudriñarnos, se asoman al fondo recóndito del espíritu de nuestros artistas llenos del afán de no pintar ya más panderetas para la exportación consagrándose, en cambio, con cariño efusivo y comprensivo, a difundir la legítima representación del alma andaluza y a proyectar su verdadera luz sobre todos los prejuicios y todas las falsas interpretaciones.

La singular índole desinteresada de este certamen anual es una cualidad precisa y característica de nuestras exhibiciones de arte.

Aqui no se hacen oposiciones a recompensas, ni se libran batallas apasionadas por obtener los premios. Aquí se organiza una verdadera exposición de arte puro, donde, por lo mismo, no se concibe el análisis crítico, sino la síntesis estética:

5. Nacido en Aracena estuvo toda su vida vinculado a Sevilla en donde ejerció primero como colaborador de "El Noticiero Sevillano" y luego como redactor jefe de ABC desde su fundación. Fue cronista de la provincia de Sevilla, secretario del Patronato de Cultura y director de la Revista "Archivo Hispalense" de la Diputación provincial de Sevilla. Ejerció como director de prensa de la Exposición Iberoamericana. En 1930 obtuvo el premio Mariano de Cavia y cinco años después nombrado hijo predilecto de Aracena. En 1933 fue miembro de la Asamblea Pro Estatuto andaluz Fue autor de una extensa obra literaria de la que destaca: "Epistolario Bético"; "Misterio de dolor"; "Sevilla en flor" y "Romero junto a la ermita". 
trátase nada más que de mostrar al mundo la altísima valoración del espíritu andaluz por las noble actividad que cada año desarrollan para interpretar la belleza los artistas expositores, todos iguales ante la única categoría posible de Arte: la de hacer Arte.

Para este esfuerzo, estimulado nada más que por la melodía del gozo íntimo de cada artista, no se esperan otras recompensas que aquellas que otorguen los propios espectadores al elegir cada uno para sí una obra de arte y adquirirla por el placer imcomparable de llevarse consigo un pedazo de nuestro espíritu..." 6

En esta ocasión acuden a la muestra 67 artistas plásticos y dos ceramistas, la mayoría andaluces pero también de otras regiones españolas que presentan un total de más de 267 obras. De entre los primeros, la mayoría pintores, algunos consagrados como el Conde de Aguiar, Felipe Abárzuza, Gonzalo Bilbao, García Rodríguez, Gómez Gil, Gil Gallangos, González Santos, Federico Godoy, Lacárcel Aparici, José Lafita y Muñoz Lucena.

La temática general -como es de rigor-, aborda géneros costumbristas y paisajes, los más en boga por entonces, siendo los relativos a edificios y monumentos sevillanos, bien históricos o recién inaugurados o remozados entonces, los más cultivados; así: la Casa de Pilatos, Sta. Clara, Calle Mármoles, interior de San Martín, Plaza del Pan, vistas del Alcázar, Jardines de Murillo, Patio de los Venerables, Plaza de San Marcos, Callejón de la Judería, Calle Susona e incluso la no ha mucho terminada Plaza de América. No faltan tampoco paisajes urbanos de ciudades consideradas "emblemáticas" y dadas a conocer popularmente por el turismo, entonces incipiente pero prometedor, que querían actualizar su visión romántica de ellas, como son, entre otras, Toledo, puesta de moda por el Marqués de la Vega Inclán, a la sazón Comisario Regio de Turismo y fundador de la casa de El Greco; Granada, la siempre romántica y pintoresca ciudad de La Alhambra; Alcalá de Guadaira; Carmona y Aracena, entre otras.

El capítulo de la escultura está representado en la exposición ateneísta por obras de maestros consagrados como Sánchez Cid y de algunas jóvenes promesas como Pérez Comendador e Illanes Rodríguez quienes gustan realizar versiones esculpidas de tipos y figuras andaluces, v. g, cabezas femeninas, así como retratos.

La prensa local y regional se encarga de recoger en sus paginas el nivel artístico alcanzado en el certamen ateneísta cuya critica es en general favorable recalcando los valores de exaltación regionalista de la mayoría de las obras presentadas. ${ }^{7}$

En Junta Directiva de la Sección de Bellas Artes celebrada con posterioridad, presidida por el pintor Gustavo Bacarisas, se acuerda la manera de repartir o conceder los premios de estímulo a los jóvenes artistas atendiendo al resultado de las cuentas de la exposición, las cuales y, "según confidencia del encargado de la misma, Sr. Valdés quedaron todas cubiertas con el ingreso del tanto por ciento

6. Exposición de Bellas Artes. Catálogo. Sevilla, 1925. Pág. 7 y 8.

7. "Exposición de Bellas Artes" "El Liberal". Sevilla, 30/3/1925. Pág. 2a. "Consideraciones sobre la Exposición de pintura". "El Liberal", Sevilla, 7/5/1925. Pág. 4. 
obtenido con las ventas de los cuadros". ${ }^{8}$ De este modo, se conceden los siguientes premios: Con 175 ptas. a Miguel García; Juan José Orta; Joaquín González Sáenz; Rafael González Sáenz; José Fernández Venegas y Juan Núñez.

Con 100 ptas. a Fernando del Toro; Elías Ferrer; Enrique Pozo; Mauricio Tinoco; Miguel González Migolla; Manuel Martínez de León; Pablo Sebastián Canto y Carlos Roquete, algunos de los cuales estaban fuera de catálogo.

La vida artística del Ateneo hispalense se hallaba, como estamos viendo, en plena actividad en este año vigésimo quinto del siglo, pese a los avatares económicos por los que atravesaba la institución y de los que se hace cargo la nueva junta elegida en 29 de mayo. ${ }^{9}$ Ello se constata en algunas actas de sus frecuentes juntas generales o directivas en las que se debate tan crucial punto y la forma de hacer frente a la débil economía de la Sección de Bellas Artes. En este sentido, en una reunión de la directiva del mes de junio presidida por el pintor González Santos, se toman algunos acuerdos ante "tan precaria situación económica", debida a "los muchos recibos sin abonar, pues de los 88 de ellos atrasados solo se han cobrado unos 12, por lo que se apremia a los socios a ponerse al corriente". Por otra parte y con respecto al tema económico se acuerda "celebrar algún festival para obtener recursos ". ${ }^{10}$ En este mismo sentido, se llega incluso a opinar por algunos (Pozo, Molleja y Díaz Jara ), en abierta oposición a la directiva, que: "la Junta no ha debido repartir los premios sin conocer las cuentas definitivas". "1

No obstante esta adversa situación financiera, los miembros de la Sección de Bellas Artes del Ateneo hispalense mantienen una moral alta y combativa propia de una juventud (la mayoría de ellos estaban en la primavera de la vida) ilusionada y con ganas de triunfar, reflejo del ambiente artístico y cultural que se vivía en la noble institución pese al decaimiento general que se observa en la ciudad, cuyo conocimiento nos llega de la mano del reputado pintor y activo ateneísta José Rico Cejudo (1864-1939) quien por entonces dirigió a su amigo y también reconocido pintor Ricardo López Cabrera (1864-1950) una "carta abierta" en la prensa local, lamentándose de que "desde hace muchos años los negocios del arte en nuestra ciudad, desgraciadamente, son negativos, pues, salvo algún que otro cuadro que pueda comprar cualquier extranjero de los que

8. Acta de la Junta directiva de 13/6/1925. Libro de Actas. Archivo del Ateneo de Sevilla, s/p.

9. La nueva junta fue elegida de la siguiente manera:

Presidente: González Santos, 11 votos

Viceprete. : F. Lacárcel. 11 votos

Secretario: José Molleja 11 "

Tesorero: Eloy Zaragoza 23 "

Vocal de clase: Francisco Espinosa 12 votos.

"secretario : Martínez del Cid 12 votos" " Bibliotecario: Rafael González Sáenz 14 votos"

Obtuvieron votos: Santiago Martínez; Sánchez Cid; F. Mora; Gómez Gil; Pozo; Juan Miguel

Sánchez; A. Grosso; Bacarisas y Miciano. Acta de la Junta Gral. de 29/5/25.

10. Acta de Junta directiva de 24 de junio de 1925. Libro de Actas del Archivo del Ateneo, s/p...

11. Acta de Junta Gral. de 2/6/25. 
nos visitan durante las fiestas de Semana Santa y Feria, nuestros paisanos, triste es decirlo, no se preocupan para nada de nuestras exposiciones, $y$, muchos de ellos que por su cultura, su posición social y su capital debieran interesarse por las cosas del arte, ni visitan dichas exposiciones". ${ }^{12}$

También y en la misma línea de pensamiento y hasta de expresión el joven artista ecijano José Molleja Espinosa (1900-1977) al hacer un balance del curso 1925/26 de la Sección de Bellas Artes, a modo de epílogo, dice: "Sentimos que hallamos de consignar que las artes sevillanas viven y se desarrollan al amparo de lo que los extranjeros compran pues el inexplicable retraimiento de la clase pudiente es total. No valen cuantos esfuerzos se han hecho por ponerse más al contacto de ella. Parecía lógico que al tener abierto el Salón Permanente la afluencia de compradores de la localidad seria manifiesta. No ha sido así...

¿Quiera Dios que en venideros años tengamos que modificar nuestro desaliento producido por falta de protección y de amor al arte moderno, que muy justificado vemos existe en Sevilla, y podamos cantar victoria!" 13

Este relajamiento, apatía e incluso crisis del arte sevillano de entonces llega a afectar a una institución tan sólida como la R...l Academia de Bellas Artes, en cuyo seno se produce en este año de 1925 una situación al parecer sin precedentes. De "crisis" la llega a calificar el autor de los Apuntes para su historia, . . lien afirma que se llega a romper "la armonía y cordialidad entre sus miembros" ${ }^{14}$, pues los numerarios de su Sección de Arquitectura, Aníbal González, Juan Talavera, Gómez Millán y González Rojas, presentan su dimisión motivada por la protesta de la corporación por las nuevas edificaciones que se están ejecutando en la ciudad no acordes con sus principios estéticos en un momento, además, de verdadera preocupación por conservar el patrimonio artístico y arquitectónico y por mantener la esencia de lo propio acordes con los postulados regionalistas de los que la prensa se hace eco ampliamente ${ }^{15}$.

La noble y real institución sevillana acepta la renuncia colectiva de tan relevantes miembros corporativos en su junta de 15 de junio que continúa en la siguiente jornada. Se proponen en su lugar para ocupar sus correspondientes plazas los consiliarios Conde de Aguiar, Gonzalo Bilbao y Rodríguez Jurado.

Pero, además, la crisis académica arrastra consigo a su entonces presidente, Carlos de la Lastra, Marqués de Torrenueva quien, tal vez por compañerismo y común espíritu ante la situación planteada, presenta su renuncia, sustituyéndole el propio Gonzalo

12. PANTORBA, B. de El pintor López Cabrera. Madrid, 1966. P. 39

13. Sección de Bellas Artes del Ateneo de Sevilla. Memoria del Curso 1925/26 por el secretario de la Sección José Molleja y Espinosa. Imprenta A. Padura. Sevilla, 1926. P. 21.

14. MURO OREJÓN, A. Apuntes para la historia de la Academia de Bellas Artes de Sevilla. Sevilla, 1961. Pág. 85.

15. Sirvan de ejemplo los siguientes artículos: "La restauración de la torre de D. Fadrique", "E1 Liberal", 29/1/25. Pág. la . "La iglesia de Sta. Catalina", "El Liberal", 5/3/25. Pág. 1". "Las vidrieras de la catedral". "El Liberal", 26/5/1925. Pág. 4" y 28/5/1925, Pág. la". 
Bilbao, al tiempo que continúa de primer consiliario el Conde de Aguiar y se designan $2^{\circ}$ y $3^{\circ}$ a Rodríguez Jurado y Hazañas y la Rua, respectivamente.

Este mismo año, como hecho jubiloso, en 14 de noviembre, la Real Corporación admite en su seno a un prestigioso ateneísta y ya, aunque joven, destacado pintor: Santiago Martínez Martín, presidente de la junta directiva de la Sección de Bellas Artes el año anterior.

El Ateneo, como decíamos, se halla por entonces en plena vitalidad pese a su famélica economía a la que hace frente con sobriedad e iniciativas saludables para obtener recursos. No cesa en promover actividades proartísticas. En este sentido, mientras el presidente de la Sección de Bellas Artes anuncia en el mes de junio que "se han hecho gestiones para saber si la misma había sido invitada al certamen artístico de Cádiz en el próximo agosto para lo cual se acordó enviar colectivamente y en nombre de la misma las obras de los socios que pensaran mandar, al tiempo que en lo sucesivo este acuerdo quede como obligado sistema para concurrir a todas las exposiciones fuera de Sevilla", se pide, en el mismo mes, una de las Salas de Arte Antiguo al Comité de la Exposición Iberoamericana como salón de exposiciones permanentes de que "tan necesitados estamos". E, igualmente, en la misma ocasión el socio directivo y prometedor pintor José Martínez del Cid (1904-1986) pide que se visite al alcalde de Sevilla para que active los ejercicios de oposición para cubrir las pensiones de artistas. ${ }^{16}$

Por otra parte y también en el mes de junio en junta general de la Sección el presidente, a la sazón González Santos, da cuenta del proyecto de intercambio de la misma con un centro americano, pues había sido elegida, junto con la de Medicina, para ello. Así mismo se anuncia la invitación cursada a esta Sección por el Círculo andaluz de Buenos Aires para que concurran sus socios a exponer allí sus obras. En este sentido acude a la capital argentina el pintor Juan Miguel Sánchez. También en el mes citado, se aprueban las bases para el concurso que ha de confeccionar el sello -logotipo de la Exposición Iberoamericana. Abierto el mismo en el mes de octubre se presentan cuatro trabajos aspirantes cuyos lemas eran: "España y América en Sevilla", "Crepúsculo ", "Occidente" y "X". ${ }^{17}$ Finalmente fue en 1928 cuando su diseño correría a cargo del pintor Santiago Martínez, entonces vocal artístico del Comité de la Exposición, consistente el mismo en una iconografía muy representativa del acontecimiento, en cuyo centro aparece la Giralda, símbolo de Sevilla, sostenida por el globo terrestre en el que se distingue parte del nuevo continente tras la cual se representa una de las carabelas colombinas. Todo ello se circunscribe por su parte inferior con la leyenda"Exposición Iberoamericana". El resto lo compone una profusa decoración a base de elementos fitomorfos y frutales que se acompañan de las inscripciones "Sevilla y 1929, 1930".

16. Junta directiva de la Sección de Bellas Artes de 24/6/25. Libro de Actas. S/p.

17. Junta directiva de $8 / X / 25$. 
Durante el verano de 1925 el Ateneo fomenta el arte cinematográfico llevándolo en veladas mixtas populares a los corrales de vecinos ante multitudinario auditorio procedente de las clases sociales más humildes. Tales son las que se llevaron a cabo en algunos de los más célebres: Corral de la plaza de Mengíbar, en cuyo patio se situaron más de 600 personas; en el del Conde, de la calle Santiago; en el Corral de los Carros", en donde se proyectó una película cómica, y en el llamado de la Esperanza, para los vecinos de la Resolana. ${ }^{18}$

A fines del estío se había reunido de nuevo la junta general de la Sección ateneísta presidida en esta ocasión por el eximio pintor valenciano pero sevillanizado Félix Lacárcel, para tomar entre otros acuerdos el de la aprobación de reglamento del salón de la exposición permanente que, con la modificación del porcentaje de las ventas hechas por José Lafita, se aprueba.

En esta misma ocasión el propio presidente "propone una excursión a la interesantísima ciudad de Écija" a efectuar el último trimestre del año, siendo su coste de 35 ptas. y la estancia de dos días, aprobándose su celebración, dada entre otras razones el propio espíritu ateneísta en cuyos estatutos fundacionales figura como Sociedad de Excursiones. ${ }^{19}$

Entre los medios con que el Ateneo cuenta para el perfeccionamiento artístico y la puesta al día en materia artística de los socios de su Sección de Bellas Artes figura la invitación que con frecuencia hace a determinados artistas sevillanos y foráneos, para que expongan aquí sus obras. En este sentido en el mismo mes de septiembre y en el siguiente tenemos constancia de este hecho tanto en la junta directiva como en la general de la Sección; así, se solicita que exponga sus obras al eximio pintor Gonzalo Bilbao, comenzando de este modo la serie de exposiciones particulares de pintores españoles que se proyecta realizar, continuando con Andrés Parladé; Gustavo Bacarisas; el propio presidente entonces de la Seccion, Manuel González Santos; Eugenio Hermoso; Covarsí; Pedraza Ostos; Pérez Comendador; Pino y Sardá; Rodríguez Jaldón o Vázquez Díaz, entre otros.

Singular en este orden de exposiciones, es la que propone Lacárcel que se haga del canario Néstor en este año de 1925, para que "presente sus maravillosas obras en el salón permanente". La iniciativa del directivo ateneísta encuentra su justificación ante su, por entonces en Sevilla, novedosa obra simbolista cargada de evocaciones fantásticas, representada en su grandioso "Poema del mar", expuesto el año anterior en los salones de la Sociedad Española de Amigos del Arte de Madrid. ${ }^{20}$

En otro orden de actuaciones ateneístas y con vista a la formación artística de sus socios y de la juventud sevillana en general, figuran desde tiempo atrás las clases gratuitas de dibujos impartidas en su seno. En septiembre, se designa al profesor encargado de la misma que recae de nuevo en Francisco Luna por petición unánime

18. Pablo Romero, M. Historia del Ateneo de Sevilla. Sevilla, 1982. PP. 243 y 244.

19. Junta Gral. de $8 / \mathrm{IX} / 25$.

20. Junta Gral, de $1 / X / 25$ 
de los miembros de la junta directiva, y pese a la petición solicitada también por José Rodríguez Tamarit que queda desestimada. ${ }^{21}$

Días después, ya en el mes de octubre, se procede a la apertura del nuevo curso de la Sección en la que se da cuenta de la presentación de 18 solicitudes para ocupar diez plazas gratuitas en las clases de Dibujo. Después de verificar la oposición 16 aspirantes, las obtuvieron los siguientes, por este orden:

$1^{\circ}$ Maximiliano Cruz Rubio

$2^{\circ}$ Sebastián Santos

$3^{\circ}$ José Macias Santos

$4^{\circ}$ José Morillo Fernández

$5^{\circ}$ Eduardo Acosta

$6^{\circ}$ Francisco Hidalgo

$7^{\circ}$ José Moreno Rodríguez

$8^{\circ}$ José María Sanchiz Troyano

$9^{\circ}$ Joaquín Moreno Moreno

$10^{\circ}$ José Díaz

Al propio tiempo quedaron aprobados para ocupar las vacantes que se produjeran:

$1^{\circ}$ Julio Atienza

$2^{\circ}$ Enrique Estela Antón

$3^{\circ}$ Francisco Bernal Ríos

$4^{\circ}$ Enrique Bolaños

$5^{\circ}$ Eustaquio Marín ${ }^{22}$

Como complemento a la labor artística desarrollada por el Ateneo hispalense en este año de 1925, a través de su Sección correspondiente en la que no está ausente la pedagógica llevada a cabo en las clases gratuitas de Dibujo práctico de talleres por el escultor Adolfo López, hay que señalar también la que podríamos llamar de erudición; esto es, los numerosos actos desarrollados en su seno correspondientes a las conferencias sobre Arte; $\mathrm{e}$ incluso las no menos interesantes actividades de la Sección de Música. Entre las disertaciones artísticas caben señalar, en primer lugar la impartida por el profesor Elías Tormo y Monzó (1869-1957), primer catedrático español de Historia del Arte, acerca de "Sevilla en San Petersburgo", en la cual revertió muchos de sus conocimientos sobre pintura de escuela española conservada en su espléndido museo ${ }^{23}$.

21. Junta Gral. directiva de $21 / / \mathrm{XX} / 25$

22. Junta Gral. de $8 / X / 25$.

23. Precisamente Elías Tormo fue uno de los primeros directores, además de Gómez Moreno, de la, en este año, creada extraordinaria revista "Archivo Español de Arte y Arqueología", una de las mejores dedicadas a los estudios artísticos en España cuya publicación quedó interrumpida en la Guerra Civil y reanudada tras ella con la denominación de "Archivo Español de Arte". 
La segunda de las conferencias de que se tiene constancia a través de las Memorias del Ateneo es la desarrollada por el pintor Antonio García Lozano (1876-?) que, como especialista en el tema, tituló "El arte en el jardín" ${ }^{24}$. Utilizó abundante material visual de diapositivas para mostrar variados aspectos de "las ideas estéticas de los jardines" a través de singulares ejemplos. ${ }^{25}$

Por su parte, el pintor sevillano Rico Cejudo dio lectura el 16 de mayo de su libro inédito de coplas titulado "Alma andaluza" ${ }^{26}$. Es obra llena de gracia y popularidad como acreditan las dotes de tan regionalista artista, cuyo amor por el tema no era nuevo en un momento de verdadera apoteosis del género llamado "flamenco" u "hondo", pues no en balde es la época cumbre del cantaor coriano Francisco González Sanromán, "Mazaco" que, con su peculiar personalidad impuso todo un estilo en este difícil arte. ${ }^{27}$

Finalmente, el curso 1925/26 de la Docta Casa contó con la participación enudita de su socio Juan Lafita, académico de Bellas Artes y director del Museo Arqueológico hispalense, quien disertó sobre el sugestivo tema: "El descubrimiento de la tumba de Tut-ankh-Amen", con motivo del envío a la Comisión de Monumentos histórico-artísticos de la provincia por parte del Duque de Alba de la conferencia redactada por el egiptólogo inglés H. Carter (1873-1939), Inspector General de Antigïedades.

Para concluir, debemos dejar constancia siquiera someramente, tal como se adelantó, de la actividad musical llevada a cabo por la Sección correspondiente del Ateneo, pues ello contribuyó, a no dudar, positivamente al enriquecimiento del ambiente artístico de la ciudad. Conviene reseñar muy especialmente y en primer lugar el brillante concierto dado por el maestro Joaquín Turina al que se homenajeó en su propia ciudad con ocasión de su nueva obra "La oración del torero ", escrita este mismo año para cuarteto de laudes, publicada para cuarteto de arco e interpretada casi siempre por orquesta, hermoso anticipo a su "Canto a Sevilla", compuesta dos años después.

La propia Sección de Música sintió también el grato deber este año de presentar en su seno al joven sevillano Francisco de Villalonga, discípulo del Profesor

24. Antonio García Lozano estudió en la Academia de Bellas Artes de Sevilla ampliando sus conocimientos en Paris a partir de 1892 y hasta el inicio de la $1^{\text {a }}$ Guerra Mundial. Como especialista en pintura de jardines llevó a cabo numerosas composiciones sobre arquitectura aplicada a jardinería llegando a alcanzar gran popularidad en la capital francesa por su participación en las exposiciones allí celebradas. En el Ateneo, en cuya Sección de Bellas Artes ingresó como socio en 1894 y cesó en 1920, expuso por primera vez en 1900 con su óleo "Las sevillanas" (Libro del Centro de Bellas Artes. Exposición 1900. No 127. S/p).

25. Ateneo de Sevilla. Memorias de los trabajos realizados durante los cursos de 1924 a 1925 y de 1925 a 1926, escrita por el secretario de la Sociedad Miguel García Bravo-Ferrer. Talleres de Imprenta de la Casa Velázquez. Sevilla, 1926. Pág. 9.

26. Véase nuestro trabajo: "Notas biográficas del pintor sevillano José Rico y Cejudo (1864-1939)": "Laboratorio de Arte". Sevilla, 1991. N4. Pág. 241.

27. CANTERLA, J. F. " Biografía incompleta del cantaor Mazaco". Rev. Azotea. Ayto. de Coria del Río. Nº1. Especial de Feria, 1987. Pág. 21. 
Fernando Palatín; e, igualmente, a las recién tituladas como profesoras de piano, Carolina Caro y Encarnación del Valle, primeros premios concedidos a la Docta Casa por la Sociedad sevillana de Conciertos.

Por último, el 30 de mayo la Sección cerro sus actividades con las oposiciones al premio Manuel de Falla, instituido también por la mencionada Sociedad y que fue otorgado al brillante alumno de violín Luis Lerate. ${ }^{28}$

Con estas actividades musicales sucintamente reseñadas y otras, no menos interesantes correspondientes a otras tantas teatrales, más propias de estudiar en otro trabajo, creemos haber dado una visión de conjunto en relación al Ateneo y la vida artística sevillana en 1925, que supuso un hito más en sus entonces 38 años de existencia y sobre todo de relación con la ciudad, cuyo rico y complejo espíritu cultural y artístico siempre ha representado íntimamente con generosidad, brillantez y sevillanía plenas, en vísperas, entonces, de acontecimientos tan importantes para ambos como la fundación de la revista poética "Mediodía" y, su consecuente creación, la Generación del 27.

28. Vid nota 25. Pág. 15 y 16. 


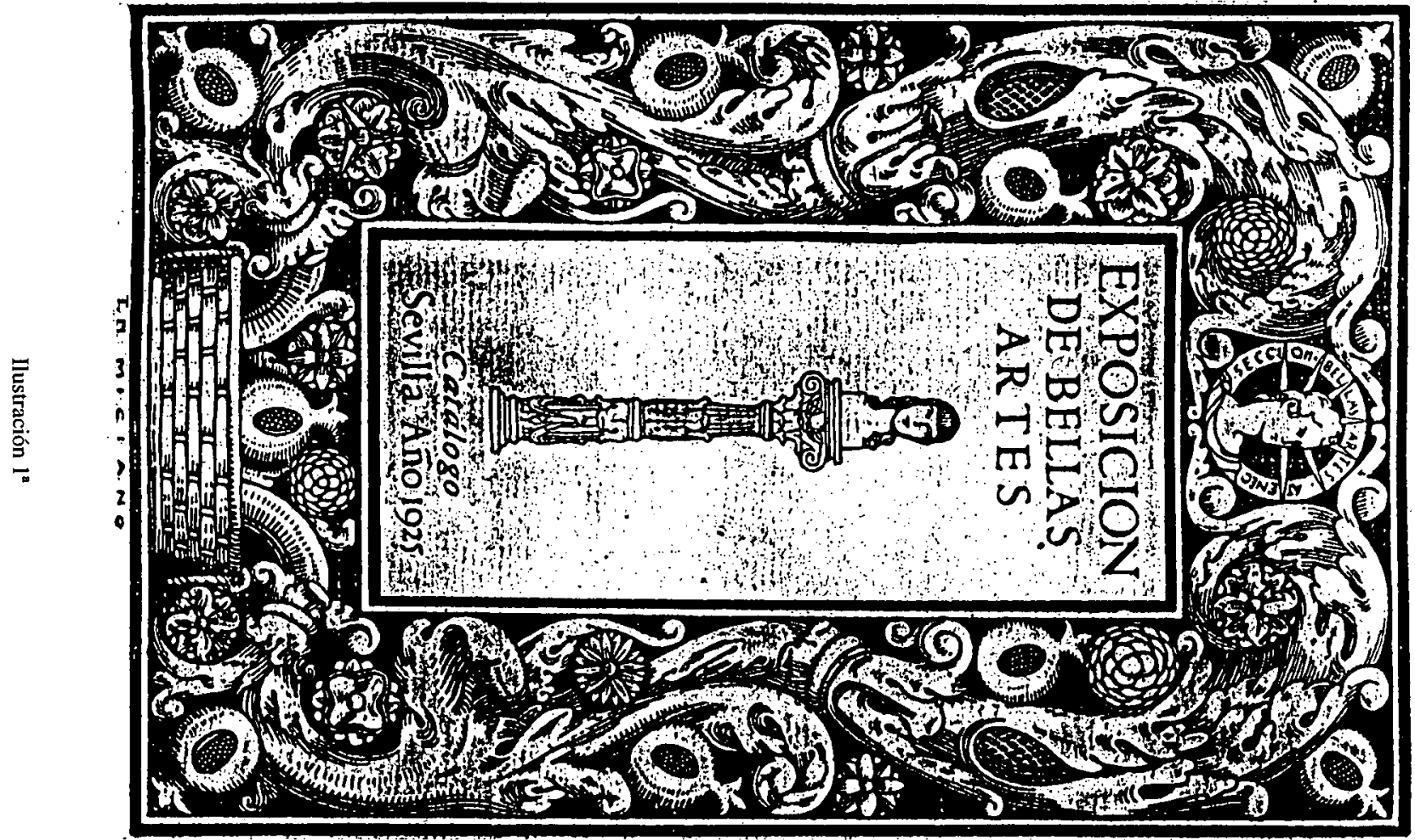




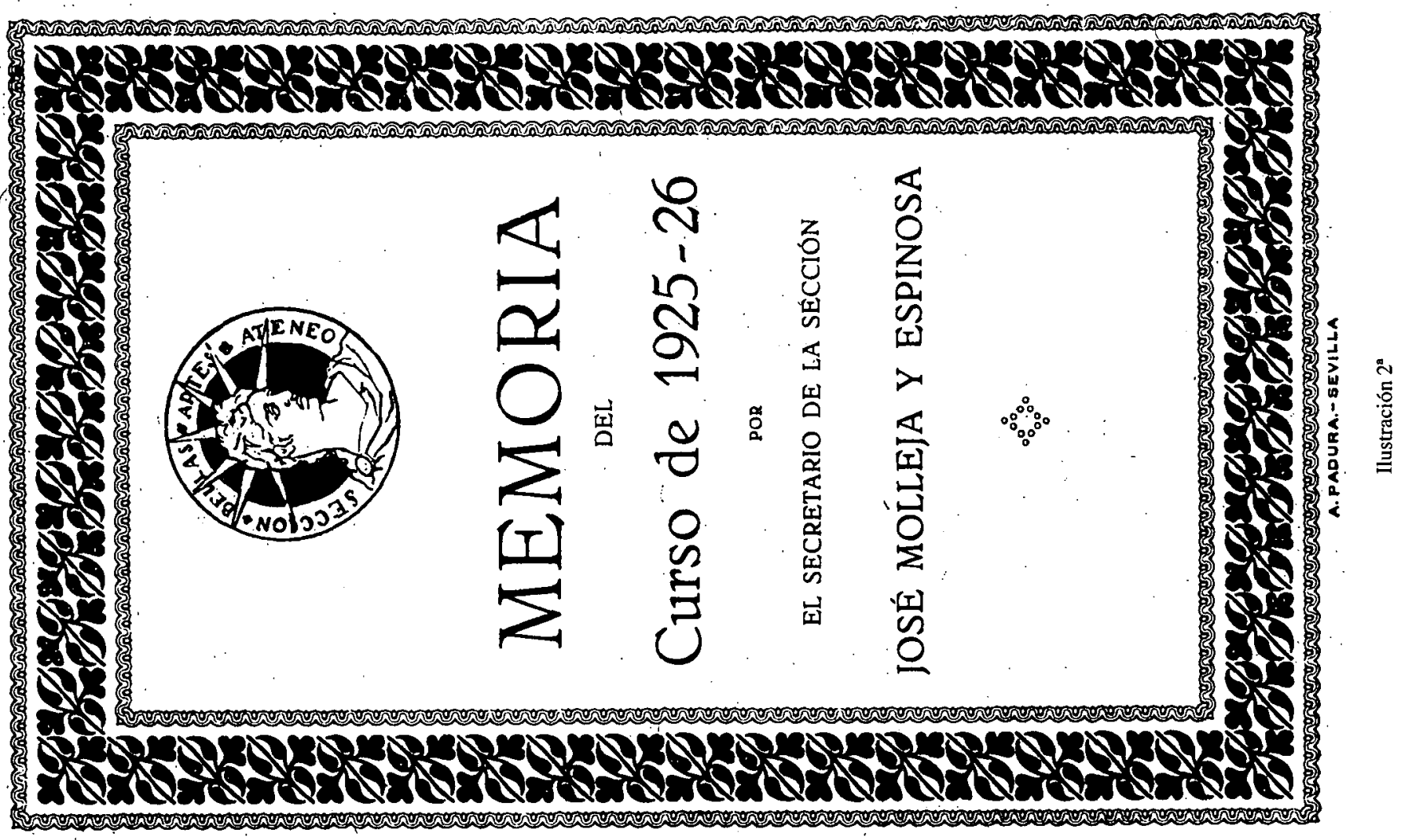






